\title{
HER-2 immunohistochemical expression as prognostic marker in high-grade T1 bladder cancer (T1G3)
}

\author{
Luca Bongiovanni $^{1}$, Vincenzo Arena ${ }^{2}$, Fabio Maria Vecchio ${ }^{2}$, Marco Racioppi ${ }^{1}$, \\ Pierfrancesco Bassi ${ }^{1}$, Francesco Pierconti ${ }^{2}$ \\ ${ }^{1}$ Department of Urology, ${ }^{2}$ Department of Pathology Catholic University of the Sacred Heart, \\ Policlinico "Agostino Gemelli", Rome, Italy
}

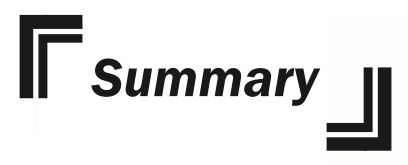

Objectives: To evaluate if the Human epidermal growth factor receptor 2 (HER-2) expression levels may be used as potential prognostic marker in high grade T1 bladder cancer (T1G3)

Methods: Specimens from transurethral resection of bladder tumour (TURBT) of 103 patients with high-grade T1 bladder cancer were collected. This pathologic database was reviewed. Four-year follow-up data were matched with pathologic data. Eighty-three patients entered the study. HER-2 staining was performed. Patients were grouped for HER-2 status. Statistical analysis included Kaplan Meier survival analysis and Log-rank test.

Results: Pathological review of TURBT specimens confirmed high-grade T1 transitional cell bladder cancer in all patients. Median follow-up was 12 months (mean 23,5; range 3-48). Twenty-one patients (25.4\%) present strong HER-2 expression (3+), 28 (33.7\%) moderate expression (2+), 26 (33.7\%) weak staining (1+) and $8(9.6 \%)$ negative expression (0). Thirtyone patients of 83 (37.4\%) had not evidence of disease, 41 (49.4\%) recurred, 11 (13.2\%) had a progression of disease. Forty-one patients had high grade T1 recurrence. Patients with HER-2 status 0 did not showed progression of disease. Patients with HER-2 status 3+, undergoing cystectomy because progression of disease, had a pathological stage > pT2 and a nodal involvement. Median Disease-Free Survival (DFS) for all patients was 12 months (DFS probability $(p D F S)=49.3 \% ; 95 \%$ CI, -11.1/+10.1). Median DFS in HER-2 groups was 8 (pDFS 37.5\%; 95\% CI,-28.8/+29.9), 24 (pDFS 46.1\%; 95\% CI,-19.5/+17.5), 20 (pDFS 46.4\%; 95\% CI,-18.8/+16.9) and 10 months ( $p D F S 47.6 \%$; 95\% CI,-21.9/+19.1) respectively in HER-2 status $0,1+, 2+, 3+$. Log-Rank test is not statistically significant $(p=0,39)$.

Conclusions: This study showed that HER-2 expression does not represent a prognostic marker of recurrence/progression of disease in high-grade T1 bladder cancer.

KEY WORDS: HER-2 expression; Prognostic marker; Bladder cancer; T1G3.

\section{INTRODUCTION}

Bladder cancer is the second most common tumour of the genito-urinary tract. In 2010, approximately 70,000 new cases of bladder cancer with almost 15,000 deaths were estimated in the USA alone (1). When the disease is first diagnosed it is non-muscle-invasive (NMIBC) in 75-80\% of cases while the remaining cases are muscle-invasive (MIBC) (2). Over 50\% of NMIBC recur, while 15-20\% advance towards a muscle-invasive form. Early diagnosis of bladder tumour improves the patient's prognosis and reduces the number of cases where cystectomy is needed. High-grade T1 lesions of the bladder (T1G3) have a high propensity to recur and progress to muscle invasion and are associated with a significant risk of metastasis and death. Long-term progression and death rates as high as $53 \%$ and $34 \%$, respectively, have been reported (3).

These bladder tumours are heterogeneous in nature and 
thus difficult to treat. Nevertheless, many of these tumours can be treated successfully with bladder preservation approaches. The dilemma facing the urologist is how best to treat these tumours in a timely manner so that the chances of bladder preservation and cancer control are maximised, while the risks of overtreatment with radical therapy are minimised (4). Useful prognostic variables and various biological makers have been proposed to assess the prognosis of bladder cancer, but the efficacy of these variables is still inadequate to accurately predict its heterogeneous behaviour. New reliable molecular indicators are required yet.

Also, during the past few decades, numerous trials have been conducted to develop new treatment regimens for both NMIBC and MIBC, because there is an urgent need to identify new agents to prevent bladder cancer recurrence and progression.

Human epidermal growth factor receptor 2 (HER-2) is a transmembrane tyrosine kinase receptor in the Epidermal Growth Factor Receptor family and it plays a fundamental role in cell growth, survival and migration. Abnormal activation of HER-2 has been proposed to lead to oncogenic transformation $(5,6)$.

Human epidermal growth factors are involved in oncogenesis through its action on several pathways leading to proliferation, angiogenesis, cell survival and metastatic potential. The role of HER-2 has been most studied in breast cancer, in which constitutively active HER-2 is overexpressed in $18-22 \%$ of cases, correlating with poor prognosis $(5,6)$. But the prognostic significance of HER2 expression status in transitional cell carcinoma (TCC) of the bladder remains uncertain. Numerous studies showed that higher HER-2 expression levels are associated with poor prognosis (7-10).

Recently HER-2 positivity was identified as an independent predictor of disease recurrence and disease specific survival in patients with TCC of the bladder after radical cystectomy (11). In contrast, other analysis showed only limited or no prognostic value of HER-2 expression (12-17).

Using Tissue MicroArray (TMA) data of 184 patients with primary TCC of the bladder, Kassouf et al. reported no significant correlation between HER-2 expression status and clinical outcomes (16). Similarly, another study reported no statistically significant difference in survival rates of 80 consecutive patients with MIBC between cases positive and normal HER-2 status (12). However, a positive HER2 protein expression status could represents a potential prognostic factor in patients affected by TCC of the bladder, particularly in high-grade $\mathrm{Tl}$ lesions, and it could be used as a novel target for adjuvant therapy $(18,19)$.

Thus, the purpose of our work was to evaluate HER-2 immunohistochemical (IHC) expression as prognostic marker of disease recurrence and/or progression in highgrade T1 bladder tumour (T1G3).

\section{Methods}

Patients selection, inclusion and exclusion criteria. From June 2005 to October 2006, specimens of highgrade T1 transitional cell bladder cancer were collected from 103 subjects ( 74 males and 29 females; average age 67,8 years, range 41-90) undergoing complete transurethral resection of the bladder tumour (TURBT) at Department of Urology, Catholic University of Sacred Hearth, Rome-Italy.

In January 2010 we have performed a review on this pathologic database. Our uropathologist (F.P.) reviewed bladder tumour resection specimens in order to confirm stage/grade of the bladder tumour.

The 2002 TNM classification (updated to 2009 TNM Classification) was used for pathological staging. The 2004 WHO/ISUP classification was used for pathological grading.

Then, 4-year follow-up data (clinic database) of all patients were matched with pathologic data. Inclusion criteria of the study encompassed, namely: presence of highgrade T1 transitional cell bladder cancer (established by pathological examination of complete TURBT specimens in whom muscularis propria was present and negative), all patients were first-diagnosed bladder cancer, all patients had complete 4-year follow-up clinical data.

Exclusion criteria encompassed Bacillus-CalmetteGuèrin(BCG)-treated patients following TURBT; incomplete follow-up data.

Ten patients were excluded because not first-diagnosed, 8 because lost at first follow-up, 2 because incomplete follow-up data. Eighty-three patients (67 males and 16 females, average age 69,2 years, range 45-88) of 103 entered the study.

Thus, HER-2 IHC analysis was performed. This study was carried out in accordance with the guidelines set out by the Ethics Committee and all subjects prior to participation were required to sign an informed consent form.

\section{Follow-up data}

The follow-up assessment adopted for these patients includes 3-months cystoscopy and urinary cytology for the first 2 years, then every 6 months for the following 2 years. No second TUR was done. Approximately after 2 to 4 weeks following TURBT, BCG intravesical therapy induction course was performed, followed by BCG maintenance therapy if there was not evidence of disease recurrence/progression.

\section{IHC analysis of HER-2}

Four-micron-tissue sections, prepared from a formalinfixed and paraffin-embedded representative of the tumor sample, were used (one to two conventional slides of tumor when available). After deparaffinization, rehydration and antigen retrieval in citrate buffer $(10 \mathrm{mMol}, \mathrm{pH}$ 6,1), tissue sections were stained for HER2 (A0485 policlonal antibody; 1/1500, Dako, Glostrup, Denmark). HER2 positivity was assessed using the ASCO scoring system, evaluating only membranous staining (20).

Specimen of normal breast tissue were used for negative control and invasive ductal breast carcinoma served as positive controls. The level of HER2 protein expression was assessed semiquantitatively by the intensity and percentage of staining and score on a scale of 0 to $3+$. Score of 0 and $1+$ are categorized negative, $2+$ as weakly positive, and $3+$ as strongly positive. Score 0 was defined as negative membrane staining in all neoplastic cells or 
Figure 1.

Membrane HER-2 stain intensity.

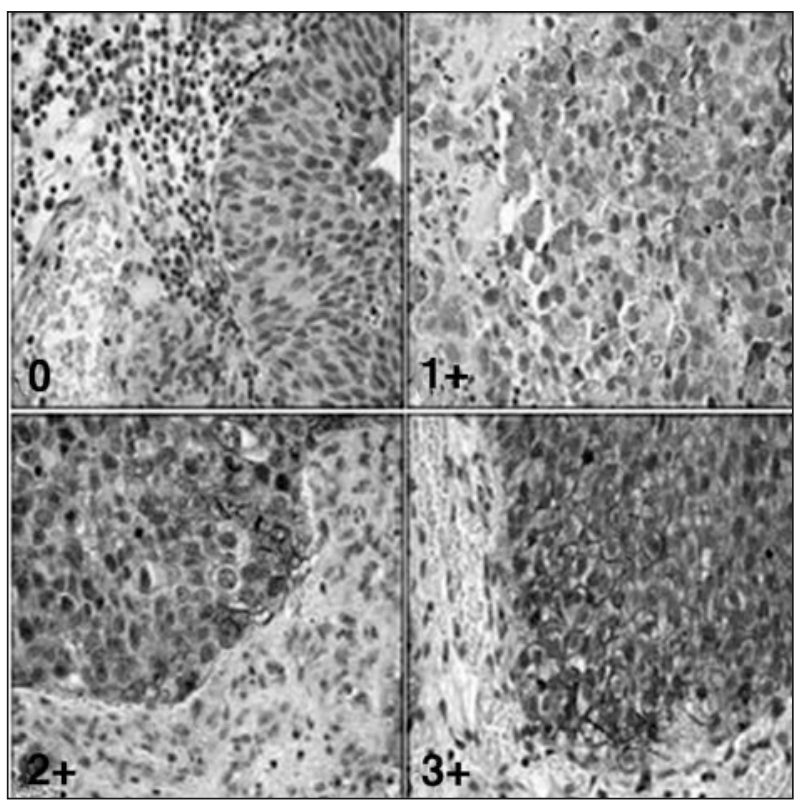

when membrane staining was observed in $<10 \%$ the tumor cells. Score 1+ was defined as faint/ barely perceptible membrane staining in $>10 \%$ of the cells and the cells exhibit incomplete membrane staining. Score $2+$ was defined weak-to-moderate complete membrane staining detected in $>10 \%$ of tumor cells. Score $3+$ was defined a strong complete membrane staining in > 10\% of tumor cells (Figure 1).

A cytoplasmic staining was considered non specific.

\section{Outcome measures and statistical analysis}

After HER-2 staining, patients were grouped for HER-2 status in 4 groups.

Kaplan-Meier survival analysis was performed to obtain survival values as Disease-Free Survival (DFS) for all patients and DFS between 4 patient groups of HER-2 status.

The difference in survival rates was determined by Logrank test. Statistical significance (p) was set at 0.05 .

Statistical tests were carried out using MedCalc Statistical Software (MedCalc Software bvba, Mariakerke - Belgium).

\section{RESULTS}

Pathological review of bladder tumour specimens confirmed high-grade T1 transitional cell bladder cancer in all patients (average diameter of lesions $2 \mathrm{~cm}$, range 13,5 cm). Median follow-up was 12 months (mean 23,5; range 3-48)

Regarding the expression of HER-2 protein, 21 patients (25.4\%) present strong expression (HER-2 score 3+), 28 (33.7\%) moderate expression (HER-2 score 2+), 26 (33.7\%) weak staining (HER-2 score 1+) and 8 (9.6\%) negative expression (HER-2 score 0 ).

Table 1 shows HER-2 status of patients (grouped in 4
Table 1.

HER-2 status of patients (grouped in 4 groups) and its matching with follow-up data (no evidence of disease (NED), recurrence (REC) and progression (PROG) data).

\begin{tabular}{|lllll|}
\hline HER-2 status & $\mathbf{N}(\%)$ & NED (\%) & REC (\%) & PROG (\%) \\
\hline$(0)$ & $8(9.6)$ & $1(3.2)$ & $7(17.2)$ & $0(0)$ \\
\hline$(1+)$ & $26(31.3)$ & $11(35.5)$ & $11(26.8)$ & $4(36.4)$ \\
\hline$(2+)$ & $28(33.7)$ & $12(38.7)$ & $13(31.7)$ & $3(27.2)$ \\
\hline$(3+)$ & $21(25.4)$ & $7(22.6)$ & $10(24.3)$ & $4(36.4)$ \\
\hline Total & $\mathbf{8 3 ( 1 0 0 )}$ & $\begin{array}{l}\mathbf{3 1 ( 1 0 0 )} \\
\mathbf{( 3 7 . 4 )}\end{array}$ & $\mathbf{4 1 ( 1 0 0 )}$ & $\mathbf{1 1 ( 1 0 0 )}$ \\
\hline
\end{tabular}

Table 2.

HER-2 status and its association with pathological examination of cystectomy specimens.

\begin{tabular}{|lll|}
\hline HER-2 status & PROG (\%) & $\begin{array}{l}\text { Pathological examination } \\
\text { of cystectomy specimens }\end{array}$ \\
\hline$(0)$ & $0(0)$ & No cystectomy specimens \\
\hline$(1+)$ & $4(36.4)$ & $\begin{array}{l}3 \text { (pT2a pNO pMx G3) } \\
1 \text { (pT3a pN1 pMx G3) }\end{array}$ \\
\hline$(2+)$ & $3(27.2)$ & $\begin{array}{l}2(\mathrm{pT} 2 \mathrm{~b} \text { pNO pMx G3) } \\
1 \text { (pT3a pN1 pMx G3) }\end{array}$ \\
\hline$(3+)$ & $4(36.4)$ & 2 (pT3a pN1 pMx G3) \\
& & 1 (pT3a pN2 pMx G3) \\
& & \\
\hline Total & $\mathbf{1 1}(\mathbf{1 0 0})$ & \\
\hline
\end{tabular}

groups) and its matching with follow-up data (no evidence of disease (NED), recurrence (REC) and progression (PROG) data).

Please note that 31 patients of $83(37.4 \%)$ had not evidence of disease, 41 (49.4\%) recurred, 11 (13.2\%) had a progression of disease. Thus, 41 patients had high-grade T1 recurrence, 8 patients of whom with an association of carcinoma in situ of the bladder (CIS).

Eleven patients of 83 showed progression from NMIBC to MIBC, requiring cystectomy. HER-2 status and its relative association with pathological examination of cystectomy specimens is reported in Table 2.

High-grade T1 lesions with HER-2 status 0 did not showed progression of disease. Interestingly, all patients with HER-2 status 3+, undergoing cystectomy because progression of disease, had a pathological stage > pT2 and a nodal involvement.

Figure 2 and 3 shows the Kaplan Meier plots of DFS for all patients and DFS between the 4 patient groups of HER-2 status.

Median DFS for all patients was 12 months (DFS probability $(\mathrm{pDFS})=49.3 \%$; 95\% CI, $-11.1 /+10.1)$. Median DFS in HER-2 groups was respectively:

- HER-2 status $0=8$ months (pDFS 37.5\%; 95\% CI, $-28.8 /+29.9)$;

- HER-2 status 1+= 24 months (pDFS 46.1\%; 95\% CI, $-19.5 /+17.5)$; 
- HER-2 status 2+ = 20 months (pFS 46.4\%; 95\% CI $-18.8 /+16.9)$;

- HER-2 status 3+ = 10 months (pDFS 47.6\%; 95\% CI, $-21.9 /+19.1)$

Log-Rank test was not statistically significant $(p=0,39)$.

Figure 2.

Kaplan Meier plot of disease-free survival (\%).

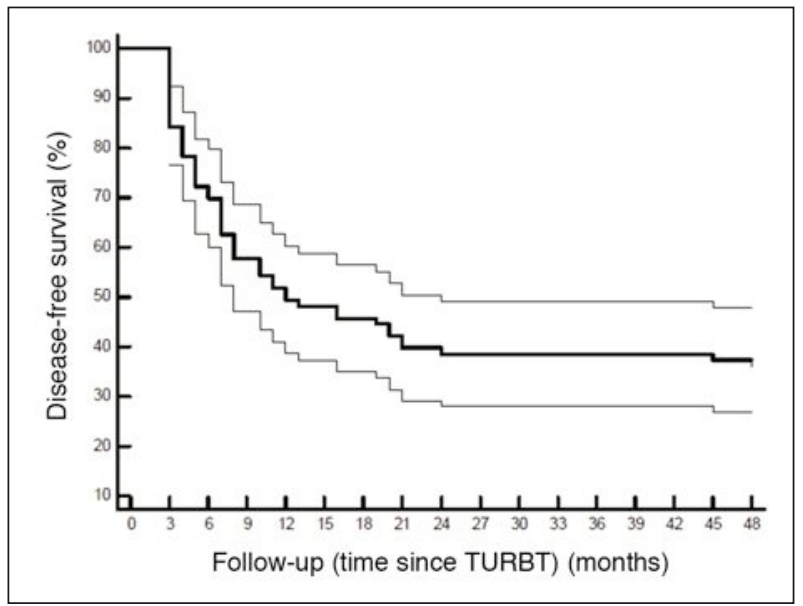

Figure 3.

Kaplan Meier plot of disease-free survival (\%) for groups of HER-2 status.

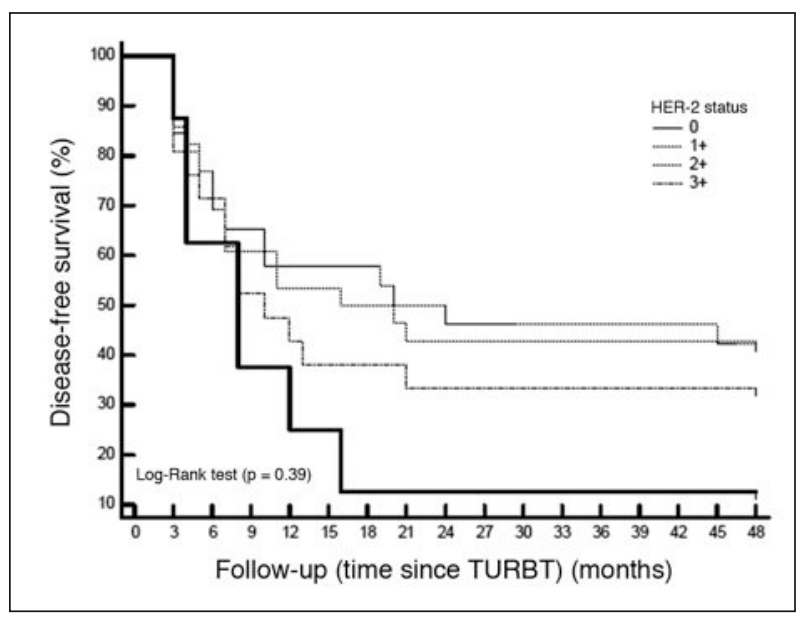

\section{Discussion}

At each stage of bladder cancer, clinical management strategies are aimed at preventing disease recurrence/progression and the use of unnecessary and potentially lifealtering procedures.

Once the disease becomes muscle-invasive, the main goal of treatment is threefold: to maximize long-term survival, to prevent pelvic recurrence or metastases, and to provide a good quality of life (21).

General guidelines exist for treatment of high-risk TCC of the bladder $(22,23)$.

However, to predict exactly which patients will progress, and who could, therefore, require more aggressive therapy, needs an individualized approach, although assessment remains more an art than science (24).

Zhau et al. reported HER2 amplification and overexpression in bladder cancer for the first time in 1990 (25).

In contrast with its known importance in breast cancer, the significance of HER2 expression and/or HER-2 gene amplification in bladder cancer is controversial. It was found that HER2 is overexpressed with a greater frequency in higher grades (40\%) and stages (38\%) than in lower grades (0\%) and stages (8\%)[8] and several studies confirmed that HER-2 could have a role as prognostic factor in bladder cancer, correlating its overexpression with poor prognosis for patients (shorter median survival time, reduced complete response to chemoradiation therapy) (8, 10, 13, 26-29).

Recently Bolenz et al. identify HER-2 positivity as an independent predictor of disease recurrence and specific survival in patients TCC of the bladder after radical cystectomy (11).

In literature other data seem to indicate limited or no prognostic value of HER-2 expression (12, 14-17).

In a large series of patients with primary TCC of the bladder, no significant correlation between HER-2 expression status and clinical outcome it has been reported (15).

Moreover, no statistically significant difference in the survival rates of 80 consecutive patients with MIBC has been observed (14).

A recent study showed that, in a large series of transurethral resection and cystectomy (1005 cases), $5,1 \%$ of MIBC had a HER-2 gene amplification with complete concordance (100\%) between IHC and Fluorescence in situ Hybridization (FISH) analyses (30). These variations in results are due to the heterogeneity of studies with respect to kits and type of antibodies used for IHC analysis, protocols, stage of the disease studied (nonmuscle-invasive vs muscle-invasive), definition of HER-2 positivity and the material studied (fresh/formalin fixed). Thus, discordant results reported in the literature highlight a need for standardized laboratory methods.

In our work we evaluate HER-2 IHC expression as prognostic marker of disease recurrence and/or progression in high-grade $\mathrm{Tl}$ bladder tumour (T1G3)

High grade T1 lesions with HER-2 status 0 did not showed progression of disease. Interestingly, all patients with HER-2 status 3+, undergoing cystectomy because progression of disease, had a pathological stage > pT2 and a nodal involvement.

No statistically significant association between HER-2 IHC expression and recurrence/progression of disease it has been found.

\section{Conclusions}

This study showed that HER-2 expression does not represent a prognostic marker of recurrence/progression of disease in high-grade $\mathrm{Tl}$ bladder cancer.

The numbers of this cohort are actually quite small and they could affect the significance of statistical analysis. Further studies analyzing a large group of disease progression are needed. 


\section{REFERENCES}

1. Jemal A, Siegel R, Xu J, et al. Cancer statistics, 2010. CA Cancer J Clin. 2010; 60:277-300.

2. Malkowicz SB, Van Poppel H, Mickisch G, et al. Muscle-invasive urothelial carcinoma of the bladder. Urology. 2007; 69(1 Suppl):3-16.

3. Cookson MS, Herr HW, Zhang ZF, et al. The treated natural history of high risk superficial bladder cancer: 15-year outcome. J Urol. 1997; 158:62-67.

4. Kulkarni GS, Hakenberg OW, Gschwend JE, et al. An updated critical analysis of the treatment strategy for newly diagnosed high-grade T1 (previously T1G3) bladder cancer. Eur Urol. 2010; 57:60-70.

5. Olayioye MA, Neve RM, Lane HA, et al. The ErbB signaling network: receptor heterodimerization in development and cancer. EMBO J. 2000; 19:3159.

6. Yarden Y. Biology of HER2 and its importance in breast cancer. Oncology. 2001; 61 (suppl.2):1.

7.Sato K, Moriyama M, Mori S, et al. An immunohistologic evaluation of C-erbB-2 gene product in patients with urinary bladder carcinoma. Cancer. 1992; 70: 2493

8. Kruger S, Weitsch $G$, Buttner $H$, et al. Overexpression of c-erbB2 oncoprotein in muscle-invasive bladder carcinoma: relationship with gene amplification, clinicopathological parameters and prognostic outcome. Int J Oncol. 2002; 21:981.

9. Lipponen P, Eskelinen M, Syrjanen S, et al. Use of immunohistochemically demonstrated c-erb B-2 oncoprotein expression as a prognostic factor in transitional cell carcinoma of the urinary bladder. Eur Urol 1991; 20:238.

10. Kolla SB, Seth A, Singh MK, et al. Prognostic significance of Her $2 / n e u$ overexpression in patients with muscle invasive urinary bladder cancer treated with radical cystectomy. Int Urol Nephrol. 2008; 40:3219.

11. Bolenz C, Shariat S, Karakiewicz P, et al. Human epidermal growth factor receptor 2 expression status provides independent prognostic information in patients with urothelial carcinoma of the urinary bladder. BJU 2010; 106:1216.

12. Mellon JK, Lunec J, Wright C, et al. C-erbB-2 in bladder cancer: molecular biology, correlation with epidermal growth factor receptors and prognostic value. J Urol. 1996; 155:321.

13. Jimenez RE, Hussain M, Bianco FJ, et al. Her-2/neu overexpression in muscle-invasive urothelial carcinoma of the bladder: prognostic significance and comparative analysis in primary and metastatic tumors. Clin Cancer Res. 2001; 7:2440.

14. Liedberg F, Anderson H, Chebil G, et al. Tissue microarray based analysis of prognostic markers in invasive bladder cancer: much effort to no avail? Urol Oncol. 2008; 26:17.

15. Chow NH, Chan SH, Tzai T, et al. Expression profiles of ErbB family receptors and prognosis in primary transitional cell carcinoma of the urinary bladder. Clin Cancer Res. 2001; 7:1957.

16. Kassouf W, Black PC, Tuziak T, et al. Distinctive expression pattern of ErbB family receptors signifies an aggressive variant of bladder cancer. J Urol. 2008; 179:353.

17. Lonn U, Lonn S, Friberg S, et al. Prognostic value of amplification of c-erb-B2 in bladder carcinoma. Clin Cancer Res. 1995; 1:1189

18. Bellmunt J, Albiol S, Suarez C, et al. Optimizing therapeutic strategies in advanced bladder cancer: update on chemotherapy and the role of targeted agents. Crit Rev Oncol Hematol. 2009; 69:211.
19. Shariat SF, Karam JA, Lerner SP. Molecular markers in bladder cancer. Curr Opin Urol. 2008; 18:1.

20. Wolff AC, Hammond ME, Schwartz JN, et al. American Society of Clinical Oncology/College of American Pathologists guideline recommendations for human epidermal growth factor receptor 2 testing in breast cancer. J Clin Oncol. 2007; 25:118.

21. Stein JP, Lieskovsky G, Cote R, et al. Radical cystectomy in the treatment of invasive bladder cancer: long-term results in 1,054 patients. J Clin Oncol. 2001; 19:666-675.

22.Stenzl A, Cowan NC, De Santis M, et al. Treatment of muscleinvasive and metastatic bladder cancer: update of the EAU guidelines. Eur Urol. 2011; 59:1009-1018.

23. Hall MC, Chang SS, Dalbagni G, et al. Guideline for the management of non-muscle-invasive bladder cancer (stages Ta, T1, and Tis): 2007 update. J Urol. 2007; 178:2314-2330.

24. Chang SS, Cookson MS. Non-muscle-invasive bladder cancer: the role of radical cystectomy. Urology. 2005; 66:917-922.

25. Zhau HE, Zhang X, von Eschenbach AC, et al. Amplification and expression of the c-erb B2/neu protooncogene in human bladder cancer. Mol Carcinog. 1990; 3:254-25.

26. Gandour Edward R, Lara PN Jr, Folkins AK, et al. Does HER2/neu expression provide prognostic information in patients with advanced urothelial carcinoma? Cancer. 2002; 95:1009.

27. Wolff AC, Hammond ME, Schwartz JN, et al. American Society of Clinical Oncology/College of American Pathologists guideline recommendations for human epidermal growth factor receptor 2 testing in breast cancer. J Clin Oncol. 2007; 25:118.

28. Chakraverti A, Winter $\mathrm{K}, \mathrm{Wu} \mathrm{Cl}$, et al. Expression of the epidermal growth factor receptor and Her-2 are predictors of favorable outcome and reduced complete response rates, respectively, in patients with muscle-invading bladder cancers treated by concurrent radiation and cisplatin-based chemotherapy: a report from the Radiation Therapy Oncology Group. Int J Radiat Oncol Biol Phys. 2005; 62:209.

29. Underwood M, Bartlett J, Reeves J, et al. C-erbB-2 gene amplification: a molecular marker in recurrent bladder tumors? Cancer Res. 1995; 55:2422.

30. Laé M, Couturier J, Oudard S, et al. Assessing HER2 gene amplification as a potential target for therapy in invasive urothelial bladder cancerwith a standardized methodology: result in 1005 patients. Ann Oncol. 2010; 21:815.

\section{Correspondence}

Luca Bongiovanni, MD, PhD (Corresponding Author)

lucabongiov@yahoo.it

Vincenzo Arena, MD

Fabio Maria Vecchio, MD

Marco Racioppi, MD

Pierfrancesco Bassi, MD

Francesco Pierconti, MD, PhD

Department of Pathology

Catholic University of the Sacred Heart, Policlinico "Agostino Gemelli"

L.go F. Vito 1 - 00168 Rome, Italy 